\title{
ON A 2-KNOT GROUP WITH NONTRIVIAL CENTER
}

\author{
KatSUYUKi YoshikaWA
}

Jonathan A. Hillman asked "Must a 2-knot whose group has nontrivial center be fibered?" We will answer this question negatively.

\section{Introduction}

An $n$-knot $k$ is a locally flat submanifold of $s^{n+2}$ which is homeomorphic to $s^{n}$. The fundamental group of $s^{n+2}-\mathscr{N}(k)$ is called the group of $k$, where $N(k)$ is a tabular neighborhood of $k$ in $s^{n+2}$.

In [6], Neuwirth showed that the center of a 1-knot group is trivial or infinite cyclic. On the other hand, Hausmann and Kervaire [1] proved that any finitely generated abelian group is the center of an $n$-knot group $(n \geq 3)$. For $n=2$, the author [8] showed that there are fibered 2 -knots whose groups have the centers $I, Z, Z \oplus Z_{2}$ and $Z \oplus Z$ respectively. Moreover, in [2], Hillman investigated centers of 2-knot groups and obtained some results. In particular, he shows that if a 2-knot is fibered, then the center of its group is $1, Z, Z \oplus z_{2}$ or $Z \oplus Z$, and he asks if a 2-knot whose group has nontrivial center must be fibered. In this paper we will answer his question negatively. That is:

THEOREM. There exists a 2-knot which is not fibered and whose group has nontrivial center.

Received 26 October 1981. 


\section{Preliminaries}

For an element $g$ of a group $H,\langle H: g\rangle$ denotes the factor group of $H$ by the normal closure of $g$ in $H$. The subgroup of $H$ generated by a subset $S$ of $H$ will be denoted by $g p(S)$.

Let $K_{1}$ be a 2-knot and $V_{1}$ a tubular neighborhood of $K_{1}$. Let $V$ be a tubular neighborhood of a trivial 2-knot and $h: V \rightarrow V_{1}$ a homeomorphism of $V$ onto $V_{1}$. Let $K_{2}$ be a 2-knot contained in the interior of $V$. Then we obtain a 2-knot $K=h\left(K_{2}\right)$ (cf. [7]).

We will calculate the group $G$ of $K$ by the van Kampen theorem. Let $V_{2}$ be a tubular neighborhood of $K_{2}$ in $S^{4}$ which is contained in the interior of $V$ and let $G_{i}$ be the group of $K_{i}(i=1,2)$; that is, $G_{i}=\pi_{I}\left(S^{4}-{ }^{\circ}\right)$. From the definition of $K$, we have the following commutative diagram of homomorphisms induced by inclusions:

(1) $G_{1}=\pi_{1}\left(S^{4}-\stackrel{\circ}{V_{1}}\right)$
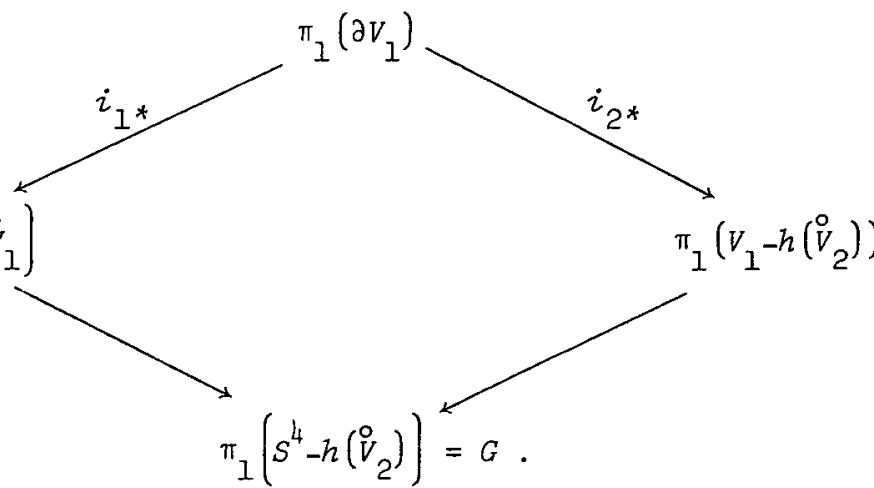

Furthermore, it is easy to see that the inclusion $j$ of $V-\stackrel{o}{V}$ into $S^{4}-\stackrel{\circ}{V}_{2}$ induces the isomorphism $j_{*}$ of $\pi_{1}\left(V-\stackrel{\circ}{V}_{2}\right)$ onto $G_{2}$. Therefore, we get the diagram, (2), of isomorphisms:

$$
\pi_{1}\left(V_{1}-h\left(\stackrel{\circ}{V}_{2}\right)\right) \stackrel{\left(h \mid V-\stackrel{\circ}{V}_{2}\right)_{*}}{\cong} \pi_{1}(V-\stackrel{\circ}{=}) \stackrel{j_{2}}{\stackrel{j_{*}}{\leftrightarrows}} G_{2} .
$$

Put $y=i_{1 *}(\tilde{y})$ and $c=j_{*}\left(h \mid V-\stackrel{\circ}{2}_{2}\right)_{*}^{-1} i_{2 *}(\tilde{y})$, where $\tilde{y}$ is a generator of the infinite cyclic group $\pi_{1}\left(\partial V_{1}\right)$. Then, from diagrams (I) 
and (2), we obtain $G=\left\langle G_{1} * G_{2}: y c^{-1}\right\rangle$.

Let $\mu$ be the order of $c$ in $G_{2}$ (if it is infinite, then put $\mu=0$ ) and let $\tilde{G}_{1}=\left\langle G_{1}: y^{\mu}\right\rangle$. Then $y$ has the order $\mu$ in $\tilde{G}_{1}$. Thus it follows that $G$ is a free product of $\tilde{G}_{1}$ and $G_{2}$ with subgroups $\mathrm{gp}(y)$ and $\mathrm{gp}(c)$ amalgamated under the mapping $y \rightarrow c$.

LEMMA. Suppose that $G_{2}$ is not infinite cyclic. If

$c(\neq 1) \in\left[G_{2}, G_{2}\right]$ and $\tilde{G}_{1} \neq Z_{\mu}$, then the commutator subgroup $[G, G]$ is not finitely generated.

Proof. To complete the proof, we use the subgroup theorem for a free product with an amalgamated subgroup [3, Theorem 5]. Let generating systems of $\tilde{G}_{1}$ and $G_{2}$ be $\alpha_{-}$and $B$-generating systems in [3], respectively. Let $x$ be an element of $G_{2}$ mapped on a generator of $G_{2} /\left[G_{2}, G_{2}\right]$ by abelianization. We choose $\left\{x^{s}: s=0, \pm 1, \ldots\right\}$ as $\alpha$ and B-representative systems for a compatible regular extended Schreier system for $G \bmod [G, G]$ (see [3]). Then the associated $\alpha$ - and $\beta$-double coset representative systems $\left\{D_{\alpha}\right\},\left\{D_{\beta}\right\}$ for $G \bmod \left([G, G], \tilde{G}_{1}\right)$ and $G \bmod \left([G, G], G_{2}\right)$ are $\left\{x^{s}: s=0, \pm 1, \ldots\right\}$ and $\{1\}$ respectively, and the $v$-double coset representatives $\left\{D_{\beta} E_{v}\right\}$ for $G \bmod ([G, G], \operatorname{gp}(c))$ are $\left\{x^{s}: s=0, \pm 1, \ldots\right\}$. Therefore, in Theorem 5 of [3], there is no t-symbol. Moreover, since

$y=c \in\left[G_{2}, G_{2}\right] \subset[G, G]$, it follows that $x^{s} \tilde{G}_{1} x^{-s} \subset[G, G]$ for each $s$. Hence, from theorem $5,[G, G]$ is a tree product of an infinite number of factors $\left\{\left[G_{2}, G_{2}\right], x^{s} G_{1} x^{-s}, s=0, \pm 1, \ldots\right\}$ with the subgroups $x_{\mathrm{gp}}^{s}(c) x^{-s}$ and $x^{s} \mathrm{gp}(y) x^{-s}$ amalgamated under the mapping $x^{s} c x^{-s}+x^{s} y x^{-s}$

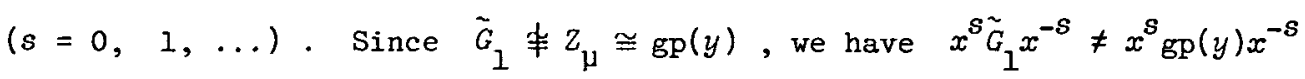
for each $s$. Hence, by $[4$, p. 53], $[G, G]$ is not finitely generated. 


\section{Proof of theorem}

We will give two examples. One has center $Z$ and the other has center $Z_{2}$. We note that the latter can not be realized as a center of any fibered 2-knot group.

EXAMPLE 1. Let $K_{1}$ and $K_{2}$ be the 2- and 6-twist-spun 2-knots of the trefoil respectively [9]. Then we have

$$
G_{1}=\left\langle y, d: y d y^{-1}=d^{-1}, d^{3}\right\rangle
$$

and

$$
G_{2}=\left\langle x, a, b: x a x^{-1}=b, x b x^{-1}=a^{-1} b,[[a, b], a],[[a, b], b]\right\rangle
$$

$[8]$.

Let $V_{i}$ be a tubular neighborhood of $k_{i}(i=1,2)$ in $s^{4}$. Let $C$ be a simple closed curve in $S^{4}-V_{2}$ which represents an element $c=[a, b]$ of $G_{2}$ and $N$ a tubular neighborhood of $C$ in $S^{4}$ such that $N \cap V_{2}=\emptyset$. Then, since $N$ is homeomorphic to $S^{1} \times B^{3}$, the manifold $S^{4}-\stackrel{\circ}{N} S^{2} \times B^{2}$ is considered as a tubular neighborhood of a trivial 2-knot in $S^{4}$. Therefore, in the previous section, we can take $V=S^{4}-R$. Let $h: V \rightarrow V_{1}$ be a homeomorphism of $V$ onto $V_{1}$ such that $j_{*}\left(h \mid V-\stackrel{Q}{V}_{2}\right)_{*}^{-1} i_{2^{*}}(\tilde{y})=c$ for a generator $\tilde{y}$ of $\pi_{1}\left(\partial V_{1}\right)$ with $i_{1}(\tilde{y})=y$. Then, from Section 2, we obtain a 2-knot $K=h\left(K_{2}\right)$ with the group $G=\left\langle\tilde{G}_{1} * G_{2}: y c^{-1}\right\rangle$.

The element $c$ has infinite order in $G_{2}$. Therefore, we have $\tilde{G}_{1}=G_{1}$. Thus $G$ is a free product of $G_{1}$ and $G_{2}$ with amalgamated subgroups $g p(y)$ and $g p(c)$. Hence, by [5, p. 211], the center of $G$ is $\operatorname{gp}(y) \cap C\left(G_{1}\right) \cap C\left(G_{2}\right)$, where $C\left(G_{i}\right)$ is the center of $G_{i}(i=1,2)$. Consequently, $G$ has the non-trivial center $\operatorname{gp}\left(y^{2}\right) \cong Z$ because 
$C\left(G_{1}\right)=\operatorname{gp}\left(y^{2}\right)$ and $C\left(G_{2}\right)=\operatorname{gp}\left(x^{6}, c\right) \quad[8]$

Furthermore, by virtue of the lemma, it follows that $K$ is not fibered.

EXAMPLE 2. Let $K_{1}$ and $K_{2}$ be the 2- and 5-twist-spun 2-knots of the trefoil, respectively. Then the group $G_{2}$ of $K_{2}$ is

$$
\left\langle x, a, b: x a x^{-1}=b, x b x^{-1}=a^{-1} b, a^{5}=(a b)^{3}=(a b a)^{2}\right\rangle,
$$

and the center $C\left(G_{2}\right)$ is $g p\left(x\left[a, b^{-1}\right],(a b a)^{2}\right) \cong z \oplus Z_{2}$ [8], [9]. We choose the element $a b a$ of $G_{2}$ as $c$. Then, in the same way as above, we can construct a 2 -knot whose group has center $Z_{2}$ and which is not fibered.

Note. Recently, T. Kanenobu communicated to the author that he has obtained another example of such a 2-knot by Fox's hyperplane cross section method.

\section{References}

[1] Jean-Claude Hausmann et Michel Kervaire, "Sur le centre des groupes de nœuds multidimensionnels", C.R. Acad. Sci. Paris Sér. A 287 $(1978), 699-702$.

[2] Jonathan A. Hillman, "Aspherical four-manifolds and the centres of two-knot groups", preprint.

[3] A. Karrass and D. Solitar, "The subgroups of a free product of two groups with an amalgamated subgroup", Trans. Amer. Math. Soc. 150 (1970), 227-255.

[4] A.G. Kurosh, The theory of groups, Volume 1, second English edition (translated by K.A. Hirsch. Chelsea, New York, 1960).

[5] Wilhelm Magnus, Abraham Karrass, Donald Solitar, Combinatorial group theory (Pure and Applied Mathematics, 13. Interscience [John Wiley \& Sons], New York, London, Sydney, 1966). 
[6] Lee Neuwirth, "The algebraic determination of the genus of knots", Amer. J. Math. 82 (1960), 791-798.

[7] Yaichi Shinohara, "Higher dimensional knots in tubes", Trans. Amer. Math. Soc. 161 (1971), 35-49.

[8] Katsuyuki Yoshikawa, "On 2-knot groups with the finite commutator subgroups", Math. Sem. Notes Kobe Univ. 8 (1980), 321-330.

[9] E.C. Zeeman, "Twisting spun knots", Trans. Amer. Math. Soc. 115 (1965), 471-495.

Faculty of Science,

Kwansei Gakuin University,

Nishinomiya,

Hyogo 662,

Japan. 